\title{
Intellectual property management in academic drug discovery: what are the challenges?
}

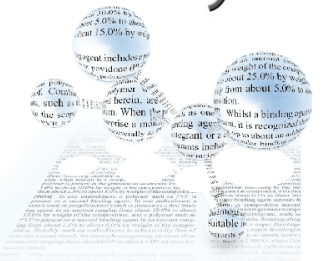

"...academic researchers should be supported by experienced drug discovery professionals and adequate budgets should be available for outsourcing critical activities."

First draft submitted: 25 November 2015; Accepted for publication: 25 November 2015; Published online: 18 February 2016

Key words: academic drug discovery • exploitation management • intellectual property management

Integrated drug discovery platforms have been established at many universities and over the last years academic drug discovery has contributed significantly to new therapies for patients [1]. The core competency of these academic platforms is generating lead compounds and demonstrating in vivo proof of concept in relevant disease models [2]. For the next step, which is testing of the novel drug candidate in expensive clinical trials, collaboration with pharma or biotech companies is sought for. Alternatively, one could try to attract venture capital and start a spinoff company to perform the clinical trials in-house. Irrespective, of the choice for one or another business model, proper protection and management of intellectual property (IP) is paramount in order to bring novel therapies to the patients. In this editorial, we want to highlight some current trends in academic IP management.

\section{Proper IP management to create value for the society}

Most academics have the noble goal of developing new therapies for unmet medical needs. However, the first priority for many of these academics is to publish their breakthrough findings immediately in highimpact journals. Indeed, publication has, and still is, an important performance metric of an academic career. As a result, there is a strong pressure to publish, which often gets in competition with the need for proper IP protection. Importantly, in the current drug development landscape it has become impossible to bring new drug candidates to the market without proper IP protection. In addition, society is increasingly expecting a return on investment of academic research. For instance for life sciences research, one is not only expecting that scientist provide novel insights, but more and more people expect that they maximally engage in translation of novel findings into new therapies for patients. In this changing landscape, academics start to better understand that proper IP management is crucial to ultimately bring a product on the market. In this respect, adequate IP management should be a natural reflex to justify the use of tax-payer's money for academic research. Although this awareness is growing, there is certainly a role for Tech Transfer Office and for Innovation Managers to continue education of academics about IP management and their moral duty.

\section{It all starts with the inventors}

An inventor is a natural person that was essential to the conception of an idea and the reduction of the idea into practice. Important is that without their personal involvement the invention would not have been devised. The correct identification of all inventors is a critical aspect for the validity

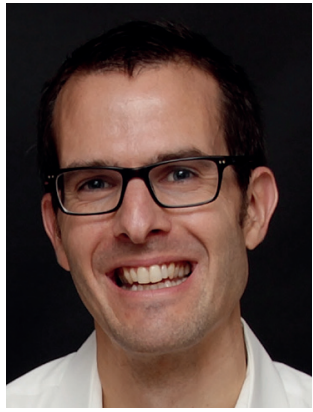

Pieter-Jan Guns

University of Antwerp, Research \& Innovation manager medical imaging, EGAMI, University of Antwerp, Antwerp, Belgium

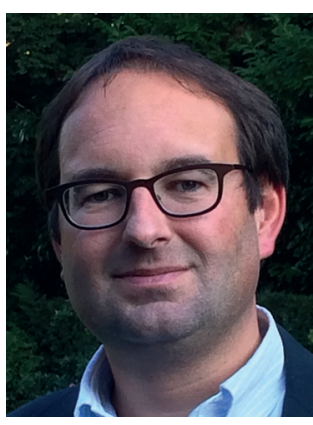

Jurgen Joossens Author for correspondence University of Antwerp, Research \& innovation manager preclinical drug discovery, ADDN, University of Antwerp, Belgium jurgen.joossens@uantwerpen.be

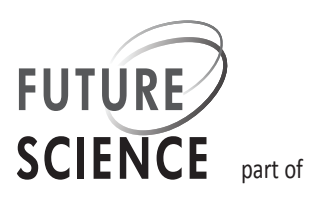


of the final patent [3]. As such, a standardized procedure of identification of the inventors is paramount. For this an 'invention disclosure document' describing the initial invention and the contribution of all stakeholders could be helpful to identify the genuine inventors. Consequently, they will receive a reward in line with regulations of their academic institution and their employment contract. Sometimes, 'noninventors' have contributed with (in-kind) resources and are not part of that rewarding policy. In such cases, it can be considered to make an additional internal agreement for the sharing of future revenue. It is evident that contributions are not solely made by internal research groups, but frequently by collaborations with different institutes. In such a case, similar agreements for revenue sharing can be set-up; however, it is advisable to formally assign one institution to take the lead in the valorization of the IP asset.

\section{Academic IP management in practice}

At the stage of deal making the value of IP is recognized by all stakeholders. However, IP management starts as of the first day of the invention when the invention has no clear nominal value yet. In short, there are two options to protect IP assets; either one opts for (temporal) secrecy or for patent filing. While, relying on confidentiality is a successful strategy in industry, keeping inventive results secret is far from evident in an academic context [4]. In drug development many years are required to further develop the IP toward a strong proof of concept. Often this period is much longer than the temporal employment contracts of some of the inventors such as PhD students and postdocs. Combined with the high publication pressure most research groups choose to protect their findings by filing a patent at an early stage. To avoid premature filing of IP, a research project ideally should be designed to combine a nonconfidential part for publication and a confidential part that could incubate for a longer time. In practice, however, inventions are mostly based on serendipity. Further, not everyone is willing to keep a part of his research secret, because they are under pressure to publish many publicly available articles to enhance their career development. It is worthwhile that the Tech Transfer Office takes the time to discuss the strategic choices and the consequences with all involved researchers. As already mentioned, it may be preferred to first further develop an IP asset based on secrecy and to patent as late as possible. Of course, this strategy can only be achieved if funding is available to prolong the contracts of essential inventors willing to do this. This again requires strategic choices, not only from the research group but also from higher management [5].
How to build successful drug development ecosystems

In the classical academic environment many IP assets do not reach the technology readiness level required by industry within an acceptable time frame due to lack of focus, missing resources, shortage in financing, absence of drug development expertise and lack of knowledge about business perspectives and market potential. For drug development, typically a $2-3$-year period with a budget of $€ 1-5$ million is needed to develop a package to convince pharma partners and/or venture capitalists. The creation of integrated academic drug discovery platforms is already an important step forward, nevertheless some development capabilities (e.g., GLP toxicology studies) or specific expertise (e.g., regulatory) are often a missing piece of the puzzle. Therefore, academic researchers should be supported by experienced drug discovery professionals and adequate budgets should be available for outsourcing critical activities. To accomplish this, a large number of incubation initiatives have been launched by universities, companies and the government [6]. Academic and governmental incubators are often just office and laboratory space that can be rented at a low fee. By contrast, industrial incubators have the additional advantage that they are close to industrial experts, but none of them deliver the needed capital to bring promising projects to clinical evaluation. Further, most incubator initiatives are organized and coordinated by one organization. It may be advantageous to join forces and to organize centralized incubators for a larger region where multiple universities, venture capitalists and companies can plug in with their projects, expertise and financial capacity. The budget gain realized by this approach could be used to finance the development of some high-potential projects. Importantly, these incubators should also support high-risk projects. Further, it is critical that the project owners have an entrepreneurial drive and understand that they do not get a free ticket. Within this ecosystem, they should be continuously challenged and project milestones need to be monitored continuously. Finally, failure of projects which is inherent to drug discovery, should not lead to frustration but rather to appreciation by the community as all stakeholders have maximally engaged to bring a new idea to the patient.

\section{Conclusion}

IP protection may not be the top priority of traditional academics. Therefore, one should offer strong incentives to inventors. In this respect, financial compensation of inventors is only one aspect. IP management and entrepreneurship could also be stimulated by 
putting more emphasis on these factors for developing an integrated academic career. More funding should be earmarked to support career development of young inventors and projects should move faster toward an incubator strategy. It is essential that the status and progress of projects is tightly monitored by scientific and business experts. If successful, these incubator projects will lead to new therapeutic products for patients. If projects fail, this should not be perceived as a complete failure as there still is an important gain for society. Indeed, these incubators strongly support entrepreneurship and simultaneously deliver welleducated drug development experts that again can sup-

\section{References}

1 Lincker H, Ziogas C, Carr M et al. Regulatory watch: where do new medicines originate from in the EU? Nat. Rev. Drug Discov. 13, 92-93 (2014).

2 Dahlin J, Inglese J, Walters M. Mitigating risk in academic preclinical drug discovery. Nat. Rev. Drug Discov. 14, 279-294 (2015).

3 Lissoni F, Montobbio F. Guest authors or ghost inventors? Inventorship and authorship attribution in academic science. Eval. Rev. 39, 19-45 (2015). port new ideas and products. It will always be a win for the society ... and it all begun with an invention that was protected by a patent application.

\section{Financial \& competing interests disclosure}

The authors have no relevant affiliations or financial involvement with any organization or entity with a financial interest in or financial conflict with the subject matter or materials discussed in the manuscript. This includes employment, consultancies, honoraria, stock ownership or options, expert testimony, grants or patents received or pending, or royalties.

No writing assistance was utilized in the production of this manuscript.

4 Frearson J, Wyatt P. Expert drug discovery in academia: the third way? Opin. Drug Discov. 5, 909-919 (2010).

5 Kelli A, Mets T, Jonsson L et al. The changing approach in academia-industry collaboration: from profit orientation to innovation support. TRAMES 17(3), 215-241 (2013).

6 Fishburn C. Translational research: the changing landscape of drug discovery. Drug Discov. Today 18(9-10), 487-494 (2013). 